Copyright by the IOP PUBLISHING LTD. Kirk T. Korista et al. 2008. "Physical Conditions in Quasar Outflows: Very Large Telescope Observations of QSO 2359-1241," ApJ 688108 doi:10.1086/592140

The Astrophysical Journal, 688:108-115, 2008 November 20

(C) 2008. The American Astronomical Society. All rights reserved. Printed in U.S.A.

\title{
PHYSICAL CONDITIONS IN QUASAR OUTFLOWS: VERY LARGE TELESCOPE OBSERVATIONS OF QSO 2359-1241 ${ }^{1}$
}

\author{
KIRK T. KORISTA \\ Department of Physics, Western Michigan University, Kalamazoo, MI 49008-5252; kirk.korista@wmich.edu \\ Manuel A. Bautista and Nahum Arav ${ }^{2}$ \\ Department of Physics, Virginia Polytechnic and State University, Blacksburg, VA 24061 \\ Maxwell Moe \\ Department of Astronomy, University of Colorado, Boulder, CO \\ Elisa Costantini \\ SRON National Institute for Space Research, Sorbonnelaan 2, 3584 CA Utrecht, Netherlands \\ AND \\ Chris BenN \\ Isaac Newton Group, Observatorio del Rogue de los Muchachos, Spain \\ Received 2008 May 12; accepted 2008 July 16
}

\begin{abstract}
We analyze the physical conditions of the outflow seen in QSO 2359-1241 (NVSS J235953-124148), based on high-resolution spectroscopic VLT observations. This object was previously studied using Keck HIRES data. The main improvement over the HIRES results is our ability to accurately determine the number density of the outflow. For the major absorption component, the populations from five different $\mathrm{Fe}$ II excited levels yield a gas density $n_{\mathrm{H}}=10^{4.4} \mathrm{~cm}^{-3}$ with less than $20 \%$ scatter. We find that the $\mathrm{Fe}$ II absorption arises from a region with roughly constant conditions and temperature greater than $9000 \mathrm{~K}$, before the ionization front where temperature and electron density drop. Further, we model the observed spectra and investigate the effects of varying gas metallicities and the spectral energy distribution of the incident ionizing radiation field. The accurately measured column densities allow us to determine the ionization parameter $\left(\log U_{\mathrm{H}} \approx-2.4\right)$ and total column density of the outflow $\left[\log N_{\mathrm{H}}\left(\mathrm{cm}^{-2}\right) \approx 20.6\right]$. Combined with the number density finding, these are stepping stones toward determining the mass flux and kinetic luminosity of the outflow, and therefore its importance to AGN feedback processes.
\end{abstract}

Subject headings: quasars: absorption lines — quasars: individual (QSO 2359-1241)

\section{INTRODUCTION}

In recent years, the potential impact of quasar outflows on their environments has become widely recognized (e.g., Silk \& Rees 1998; King 2003; Cattaneo et al. 2005; Hopkins et al. 2006). Outflows are detected as absorption troughs in quasar spectra that are blueshifted with respect to the systemic redshift of their emission line counterparts. The absorption troughs are mainly associated with UV resonance lines of various ionic species (e.g., $\mathrm{Mg}$ II $\lambda \lambda 2796.35,2803.53, \mathrm{Al}$ III $\lambda \lambda 1854.72,1862.79, \mathrm{C}$ IV $\lambda \lambda 1548.20,1550.77$, Si Iv $\lambda \lambda 1393.75,1402.77, \mathrm{~N}$ v $\lambda \lambda 1238.82$, 1242.80)

Some quasar outflows show absorption troughs from excited and metastable states. The ratio of the population level between the excited or metastable states and the ground state is sensitive to the number density and temperature of the plasma (Wampler et al. 1995; de Kool et al. 2001). Therefore, accurate measurements of the column densities associated with both excited or metastable states and the ground state of a given ion can yield the gas number density of the outflow. In addition, these measurements and similar ones of troughs from other ions and elements allow us to determine the ionization equilibrium and total column density in the outflow (Arav et al. 2001, 2007).

\footnotetext{
${ }^{1}$ Based on observations made with ESO Telescopes at the Paranal Observatories under program 078.B-0433(A).

2 Department of Astronomy, University of Colorado, Boulder, CO.
}

Accurate column densities for the outflow's troughs are difficult to determine since the outflow does not cover the emission source homogeneously (Barlow 1997; Telfer et al. 1998; Arav et al. 1997, 2003). Over the past several years, we have developed techniques for extracting reliable column densities for such situations (Arav et al. 1999a, 1999b, 2002, 2005; de Kool et al. 2001, 2002a, 2002b; Scott et al. 2004; Gabel et al. 2005). These efforts culminated with the analysis of spectroscopic Very Large Telescope (VLT) observations of the outflow seen in QSO 2359-1241 (Arav et al. 2008; hereafter Paper I). These data contain absorption troughs from five resonance $\mathrm{Fe}$ II lines, as well as those from several other metal species and metastable excited state $\mathrm{He}$ I, with a resolution of $\sim 7 \mathrm{~km} \mathrm{~s}^{-1}$ and signal-to-noise ratio per resolution element of order 100.

QSO 2359-1241 (NVSS J235953-124148; $E=15.8$ ) is an intrinsically reddened $\left(A_{V} \approx 0.5\right)$, luminous $\left(M_{B}=-28.7\right)$, radiomoderate, optically polarized $(\sim 5 \%)$, low-ionization broad absorption line quasar, at relatively low redshift $z \approx 0.868$. See Brotherton et al. (2001) for further details. Brotherton et al. (2005) describes its X-ray spectrum. An initial investigation of the physical properties of the outflow in this object using Hubble Space Telescope (HST) Faint Object Camera (FOC) and especially Keck High Resolution Echelle Spectrometer (HIRES) spectra is described in Arav et al. (2001).

The VLT spectral data set of QSO 2359-1241 is described in detail in Paper I. Its unprecedented high quality allowed us to test a variety of absorber distribution models needed to derive reliable 
ionic column densities of the outflow (see Paper I). In the present paper we report these column densities and use them to determine the physical conditions within the main component of the outflow (e, see Paper I): the ionization equilibrium, total column density and number density of the absorbing material. To do so we use the photoionization code Cloudy (Ferland et al. 1998), as well as a separate Fe II ion model (Bautista \& Pradhan 1998).

The plan of the paper is as follows. In $\S 2$ we describe the column density measurements. In $\S 3$ we determine the physical conditions in the main component of the outflow. Finally, in $\S 4$ we summarize and discuss our results and provide a simple estimate of the outflow's distance from the central continuum source.

\section{THE MEASURED COLUMN DENSITIES}

In Paper I, we presented $6.3 \mathrm{hr}$ of VLT UVES high-resolution $(R \sim 40,000)$ spectroscopic observations of QSO 2359-1241 and identified all the absorption features associated with the outflow emanating from this object. The unprecedented high signalto-noise data from five unblended troughs of $\mathrm{Fe}$ II resonance lines yielded tight constraints on outflow trough formation models.

As expected we found that the apparent optical depth model $\left[\tau_{\text {ap }} \equiv-\ln (I)\right.$, where $I$ is the residual intensity in the trough] gives a very poor fit to the data and greatly underestimates the ionic column density measurements. We found that a power-law distribution model for absorption material in front of the emission source gives a better fit to the $\mathrm{Fe}$ II data than does the standard partial covering factor model (see $\S \S 1,3,4$, and Fig. 4 of Paper I). The power-law distribution model is one in which the outflow fully covers the source, but does so inhomogeneously. This has the characteristic of allowing for nonblack saturation of absorption troughs at a large source distance ( $\sim 1 \mathrm{kpc}$; see de Kool et al. [2002c] and Arav et al. [2005] for more detailed investigations of inhomogeneous source coverage). Physically, this requires the outflow to contain many "cloudlets" with dimensions smaller than the physical span of the source. The inhomogeneous nature of the absorption also allows there to be a possibility that structure exists at the bottoms of otherwise "saturated" absorption troughs. Important for the physical conditions analysis of the present paper was the finding that the partial coverage and inhomogeneous absorption models yield similar column density estimates (see Fig. 6 of Paper I). This gives us greater confidence in the derived column density values, as they are somewhat model independent (the reasons both methods yield similar estimates are described in Paper I).

Finally, in Paper I we concluded that the power-law distribution model is more physically plausible than the partial covering model for an outflow such as this one. We thus used the power-law model as presented there to extract the column densities of all ions and ionic energy levels present in the data. For consistency we used the power-law exponent as a function of velocity of the $E=0 \mathrm{Fe}$ II lines for all other $\mathrm{Fe}$ II energy levels and for troughs from all other ions. This assumption should be robust for the $\mathrm{Fe}$ II troughs, but perhaps less so for the troughs of other ions. We direct the reader to Paper I for details concerning this column density extraction model. In Table 1 we provide the identifications for all transitions observed in outflow troughs. In Table 2 we provide the measured column densities of all species identified for the strongest absorption component, $e$ (see Paper I), many of which will be used to constrain the photoionization models, as we discuss in the next section.

\section{PHOTOIONIZATION MODELS}

\subsection{General Methodology}

The observed spectrum of QSO 2359-1241 is rich in absorption lines from singly ionized species, such as $\mathrm{Mg}$ II, $\mathrm{Si}$ II, and Ca II,
TABLE 1

Absorption Lines Identified in the VLT Spectrum of QSO 2359-1241

\begin{tabular}{|c|c|c|c|c|c|c|}
\hline$\lambda$ & $\log (g f)^{\mathrm{a}}$ & Ion & $\begin{array}{c}E_{\text {low }} \\
\left(\mathrm{cm}^{-1}\right)\end{array}$ & $g_{\text {low }}$ & $\begin{array}{c}E_{\mathrm{up}} \\
\left(\mathrm{cm}^{-1}\right)\end{array}$ & $g_{\text {up }}$ \\
\hline $2764.62 .$. & -1.95 & $\mathrm{He}^{*}$ & 159856 & 3 & 196027 & 9 \\
\hline $2829.92 \ldots$ & -1.74 & $\mathrm{He} \mathrm{I}^{*}$ & 159856 & 3 & 195193 & 9 \\
\hline 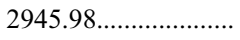 & -1.58 & $\mathrm{He}^{*}$ & 159856 & 3 & 193801 & 9 \\
\hline 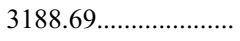 & -1.16 & $\mathrm{He}^{*}$ & 159856 & 3 & 191217 & 9 \\
\hline $3889.80 \ldots \ldots \ldots \ldots \ldots \ldots$ & -0.72 & $\mathrm{He}^{*}$ & 159856 & 3 & 185565 & 9 \\
\hline $2852.97 \ldots \ldots \ldots \ldots \ldots \ldots$ & 0.270 & $\mathrm{Mg}_{\mathrm{I}}$ & 0 & 1 & 35051 & 3 \\
\hline $2796.36 \ldots \ldots \ldots \ldots \ldots$ & 0.100 & $\mathrm{Mg}_{\text {II }}$ & 0 & 2 & 35761 & 4 \\
\hline $2803.54 \ldots \ldots$ & -0.210 & $\mathrm{Mg}_{\text {II }}$ & 0 & 2 & 35669 & 2 \\
\hline $1854.72 \ldots$ & 0.060 & $\mathrm{Al}$ III & 0 & 2 & & 4 \\
\hline $1862.79 \ldots \ldots \ldots \ldots \ldots$ & -0.240 & $\mathrm{Al}$ III & 0 & 2 & & 2 \\
\hline $1808.01 \ldots \ldots \ldots \ldots \ldots$ & -2.100 & Si II & 0 & 2 & & 4 \\
\hline 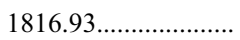 & -1.840 & Si II* & 287 & 4 & & 6 \\
\hline 3934.83 & 0.134 & & 0 & 2 & & 4 \\
\hline 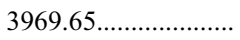 & -0.1 & & 0 & 2 & & 2 \\
\hline $2576.87 \ldots \ldots \ldots \ldots \ldots \ldots$ & 0.433 & Mn II & 0 & 7 & & 9 \\
\hline $2594.49 \ldots \ldots \ldots \ldots \ldots \ldots$ & 0.270 & Mn II & 0 & 7 & 38543 & 7 \\
\hline $2606.46 \ldots \ldots \ldots \ldots \ldots$ & 0.140 & Mn II & 0 & 7 & 38366 & 5 \\
\hline $2344.2139 \ldots \ldots \ldots \ldots \ldots$ & 0.057 & $\mathrm{Fe}_{\text {II }}$ & 0 & 10 & 42658 & 8 \\
\hline $2374.4612 \ldots \ldots \ldots \ldots \ldots$ & -0.504 & $\mathrm{Fe}$ II & 0 & 10 & 42115 & 10 \\
\hline $2382.7652 \ldots$ & 0.505 & $\mathrm{Fe}_{\text {II }}$ & 0 & 10 & 41968 & 12 \\
\hline $2586.6500 \ldots \ldots \ldots \ldots \ldots$ & -0.161 & $\mathrm{Fe}$ II & 0 & 10 & 38660 & 8 \\
\hline $2600.1729 \ldots \ldots \ldots \ldots$ & 0.378 & $\mathrm{Fe}_{\text {II }}$ & 0 & 10 & 38459 & 10 \\
\hline $2333.5156 \ldots \ldots \ldots \ldots \ldots$ & -0.206 & $\mathrm{Fe}$ II $^{*}$ & 385 & 8 & 43239 & 6 \\
\hline $2365.5518 \ldots \ldots \ldots \ldots \ldots$ & -0.402 & $\mathrm{Fe} \pi^{*}$ & 385 & 8 & 42658 & 8 \\
\hline $2389.3582 \ldots \ldots \ldots \ldots \ldots$ & -0.180 & $\mathrm{Fe} \pi^{*}$ & 385 & 8 & 42237 & 8 \\
\hline $2396.3559 \ldots$ & 0.362 & $\mathrm{Fe} \pi^{*}$ & 385 & 8 & 42115 & 10 \\
\hline $2599.1465 \ldots \ldots \ldots \ldots \ldots$ & -0.063 & $\mathrm{Fe} \pi^{*}$ & 385 & 8 & 38859 & 6 \\
\hline $2612.6542 \ldots \ldots \ldots \ldots . . . . . . .$. & 0.004 & $\mathrm{Fe} \pi^{*}$ & 385 & 8 & 38660 & 8 \\
\hline $2626.4511 \ldots \ldots \ldots \ldots \ldots$ & -0.452 & $\mathrm{Fe} \pi^{*}$ & 385 & 8 & 38459 & 10 \\
\hline $2328.1112 \ldots \ldots \ldots \ldots \ldots$ & -0.684 & $\mathrm{Fe} \pi^{*}$ & 668 & 6 & 43621 & 4 \\
\hline $2349.0223 \ldots$ & -0.269 & $\mathrm{Fe} \pi^{*}$ & 668 & 6 & 43239 & 6 \\
\hline $2381.4887 \ldots$ & -0.693 & $\mathrm{Fe} \pi^{*}$ & 668 & 6 & 42658 & 8 \\
\hline 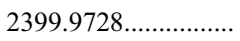 & -0.148 & $\mathrm{Fe} \pi^{*}$ & 668 & 6 & 42335 & 6 \\
\hline $2405.6186 \ldots \ldots \ldots \ldots \ldots$ & 0.152 & $\mathrm{Fe} \Pi^{*}$ & 668 & 6 & 42237 & 8 \\
\hline $2607.8664 \ldots \ldots \ldots \ldots \ldots$ & -0.150 & $\mathrm{Fe}$ & 668 & 6 & 39013 & 4 \\
\hline $2618.3991 \ldots \ldots \ldots \ldots \ldots$ & -0.519 & $\mathrm{Fe}$ & 668 & 6 & 38859 & 6 \\
\hline $2632.1081 \ldots \ldots \ldots \ldots . . .$. & -0.2 & $\mathrm{~F}$ & & 6 & & 8 \\
\hline 2338 & -0 & $\mathrm{Fe}$ & 53 & 4 & & 4 \\
\hline ................... & -0.5 & $\mathrm{Fe}$ & & 4 & & 6 \\
\hline 8 8................... & -0.983 & $\mathrm{Fe} \Pi^{*}$ & 863 & 4 & 42440 & 2 \\
\hline $2 \ldots \ldots \ldots \ldots \ldots$ & -0.228 & $\mathrm{Fe} \pi^{*}$ & 863 & 4 & 42401 & 4 \\
\hline $2411.2433 \ldots \ldots \ldots \ldots \ldots$ & -0.076 & $\mathrm{Fe} \pi^{*}$ & 863 & 4 & 42335 & 6 \\
\hline $2614.6051 \ldots \ldots \ldots \ldots \ldots$ & -0.365 & $\mathrm{Fe} \pi^{*}$ & 863 & 4 & 39109 & 2 \\
\hline 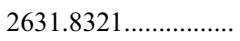 & -0.281 & $\mathrm{Fe} \pi^{*}$ & 863 & 4 & 38859 & 6 \\
\hline $2345.0011 \ldots \ldots \ldots \ldots \ldots$ & -0.514 & $\mathrm{Fe} \Pi^{*}$ & 977 & 2 & 43621 & 4 \\
\hline $2411.8023 \ldots \ldots \ldots \ldots \ldots$ & -0.377 & $\mathrm{Fe} \pi^{*}$ & 977 & 2 & 42440 & 2 \\
\hline $2414.0450 \ldots \ldots \ldots \ldots \ldots$ & -0.455 & $\mathrm{Fe} \pi^{*}$ & 977 & 2 & 42401 & 4 \\
\hline $2622.4518 \ldots \ldots \ldots \ldots \ldots$ & -0.951 & $\mathrm{Fe} \pi^{*}$ & 977 & 2 & 39109 & 2 \\
\hline 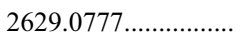 & -0.461 & $\mathrm{Fe} \pi^{*}$ & 977 & 2 & 39013 & 4 \\
\hline $2332.00 \ldots \ldots \ldots \ldots \ldots \ldots$ & -0.720 & $\mathrm{Fe} \pi^{*}$ & 1873 & 10 & 44754 & 8 \\
\hline 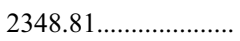 & -0.470 & $\mathrm{Fe} \Pi^{*}$ & 1873 & 10 & 44447 & 8 \\
\hline $2360.70 \ldots \ldots \ldots \ldots \ldots \ldots \ldots$ & -0.700 & $\mathrm{Fe} \pi^{*}$ & 1873 & 10 & 44233 & 10 \\
\hline $2563.30 \ldots \ldots \ldots \ldots \ldots \ldots$ & -0.050 & $\mathrm{Fe} \Pi^{*}$ & 7955 & 8 & 46967 & 6 \\
\hline $2715.22 \ldots \ldots \ldots \ldots \ldots \ldots$ & -0.440 & $\mathrm{Fe}$ II $^{*}$ & 7955 & 8 & 44785 & 6 \\
\hline $2740.36 \ldots \ldots \ldots \ldots \ldots \ldots \ldots$ & 0.240 & $\mathrm{Fe} \pi^{*}$ & 7955 & 8 & 44447 & 8 \\
\hline $2756.56 \ldots \ldots \ldots \ldots \ldots \ldots$ & 0.380 & $\mathrm{Fe} \pi^{*}$ & 7955 & 8 & 44233 & 10 \\
\hline 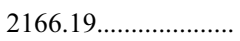 & 0.230 & $\mathrm{Ni}{ }^{*}{ }^{*}$ & 8394 & 10 & 54557 & 10 \\
\hline $2217.14 \ldots \ldots \ldots \ldots \ldots \ldots \ldots$ & 0.480 & $\mathrm{Ni} \mathrm{II}^{*}$ & 8394 & 10 & 53496 & 12 \\
\hline 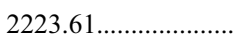 & -0.140 & $\mathrm{Ni}$ II $^{*}$ & 8394 & 10 & 53365 & 10 \\
\hline $2316.72 \ldots \ldots \ldots \ldots \ldots \ldots \ldots$ & 0.268 & $\mathrm{Ni} \Pi^{*}$ & 8394 & 10 & 51558 & 8 \\
\hline
\end{tabular}

${ }^{\text {a }}$ The $g f$ values were taken from Kurucz \& Bell (1995) for all but the Fe II lines with wavelengths given more than two decimal places. The $g f$ values for these come from Morton (2003). 
TABLE 2

Measured and Model Predicted Column Densities for the Major Outflow Trough In QSO 2359-1412

\begin{tabular}{|c|c|c|c|}
\hline \multirow[b]{2}{*}{ SPECIES } & \multirow{2}{*}{$\begin{array}{c}E \\
\left(\mathrm{~cm}^{-1}\right)\end{array}$} & \multicolumn{2}{|c|}{$\log _{10} N\left(\mathrm{~cm}^{-2}\right)$} \\
\hline & & Observed & Model $^{\mathrm{a}}$ \\
\hline 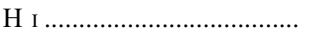 & $\ldots$ & $\ldots$ & 18.59 \\
\hline $\mathrm{He}^{*}$ & $\ldots$ & $14.14 \pm 0.03$ & 14.11 \\
\hline 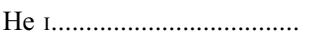 & Total & $\ldots$ & 17.14 \\
\hline 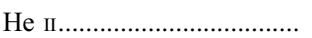 & Total & $\ldots$ & 19.46 \\
\hline 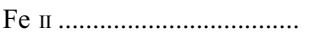 & 0 & $13.86 \pm 0.02$ & 13.84 \\
\hline Fe II & 385 & $13.51 \pm 0.04$ & 13.48 \\
\hline Fe II & 668 & $13.26 \pm 0.03$ & 13.28 \\
\hline 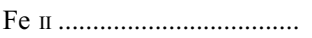 & 863 & $13.06 \pm 0.03$ & 13.08 \\
\hline Fe II & 977 & $12.85 \pm 0.03$ & 12.79 \\
\hline 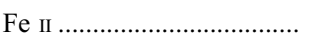 & 1873 & $13.89 \pm 0.05^{\mathrm{b}}$ & 13.83 \\
\hline Fe II & 7955 & $12.70 \pm 0.04$ & 12.67 \\
\hline 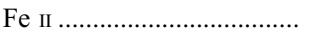 & Total & $\ldots$ & 14.41 \\
\hline 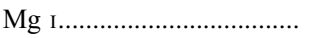 & Total & $11.92 \pm 0.03$ & 13.01 \\
\hline 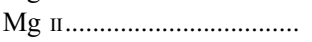 & Total & $>13.81$ & 15.22 \\
\hline $\mathrm{Si}$ II & 287 & $14.9 \pm 0.1$ & 15.19 \\
\hline 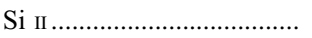 & Total & $14.9 \pm 0.1$ & 15.38 \\
\hline 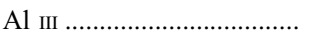 & Total & $>13.9$ & 14.35 \\
\hline 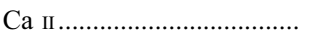 & Total & $12.52 \pm 0.03$ & 12.90 \\
\hline 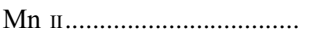 & Total & $12.71 \pm 0.03$ & 12.26 \\
\hline 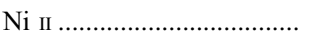 & 0 & $\ldots$ & $13.50^{\mathrm{c}}$ \\
\hline 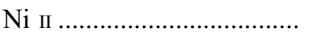 & 8394 & $12.79 \pm 0.04$ & $12.98^{\mathrm{c}}$ \\
\hline 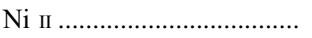 & Total & $\ldots$ & 13.70 \\
\hline
\end{tabular}

a Predicted column densities are from an optimal photoionization model with MF87 $\mathrm{SED}$, solar abundances, constant gas density $\log n_{\mathrm{H}}\left(\mathrm{cm}^{-3}\right)=4.4, \log N_{\mathrm{H}}\left(\mathrm{cm}^{-2}\right)=$ $20.556, \log U_{\mathrm{H}}=-2.418$.

b Trough partially contaminated with those of other Fe II transitions.

c Calculated from the Ni II model of Bautista (2004) assuming pure collisional excitation.

and notably from iron-peak species. We determine the physical conditions within the main absorbing component centered on $-1376 \mathrm{~km} \mathrm{~s}^{-1}$ (component $e$; see Paper I) and integrated over the range in radial velocity from -1320 to $-1453 \mathrm{~km} \mathrm{~s}^{-1}$. Our analysis of the spectrum is mostly based on the column density in the metastable $2{ }^{3} S$ excited state of He I (hereafter, $\mathrm{He} \mathrm{I}^{*}$ ) and the column densities of Fe II. For Fe II we have the level-specific column densities for the ground level $\left(a^{6} D_{9 / 2}\right)$ as well as for the excited levels $a^{6} D_{7 / 2}$ at $385 \mathrm{~cm}^{-1}, a^{6} D_{5 / 2}$ at $668 \mathrm{~cm}^{-1}, a^{6} D_{3 / 2}$ at $863 \mathrm{~cm}^{-1}$, and $a^{6} D_{1 / 2}$ at $977 \mathrm{~cm}^{-1}$ within the ground term, and the $a^{4} D_{7 / 2}$ level at $7955 \mathrm{~cm}^{-1}$ within the second excited term. The total column density in $\mathrm{Fe}$ II is estimated by scaling from the sum of the above levels (see below). These column densities as measured from our VLT spectra are presented in the upper half of Table 2. Together they constrain the physical conditions of the outflow near its hydrogen ionization front (where the bulk of $\mathrm{He} \mathrm{I}^{*}$ and $\mathrm{Fe}$ II form), as well as its total column density, as we show below. Moreover, modeling the ionization structure of these two species combined is secure because the photoionization and recombination cross sections for hydrogen and helium are well known and the ionization fraction of $\mathrm{Fe}^{+}$near the ionization front of the cloud closely tracks that of $\mathrm{H}^{+}$due to charge exchange reactions. Table 2 also lists the measured column densities of several other ions identified in the spectra.

The diagnostic power of the combined $\mathrm{He}^{*}$ and $\mathrm{Fe}$ II lines results from their different responses to temperature and density. The $\mathrm{He} \mathrm{I}^{*}$ column density depends on the population of the $2{ }^{3} \mathrm{~S}$ level of $\mathrm{He} \mathrm{I}$, which is populated by recombination from $\mathrm{He}$ II and varies with temperature, in the sense that the lower the temperature is, the higher the recombination rate is. An additional mechanism to populate the $2{ }^{3} S$ level occurs through thermalization by electronic collisions with the very long-lived $2{ }^{1} S$ level. The main depopulation mechanisms of the $2{ }^{3} S$ level are magnetic dipole radiative decay to the ground level and electron impact excitation to the neighboring $2{ }^{1} S$ (the dominant channel) and $2{ }^{3} P$ levels. For $T<15,000 \mathrm{~K}$, collisional ionization can be neglected as a depopulation mechanism of this level (see Clegg 1987). The net result is that the population of the $2{ }^{3} S$ level becomes nearly independent of density for electron densities substantially above the critical density $\approx(3-4) \times 10^{3} \mathrm{~cm}^{-3}$ (Osterbrock \& Ferland 2006; see also Arav et al. 2001). The column density in $\mathrm{He}^{*}$ is then set by that in He II, which in turn is set by the ionization parameter for a fixed spectral energy distribution of the incident continuum.

The Fe II lines in our spectrum arise from the ground and first excited multiplets of the ion. The populations of these levels are dominated by electron impact excitation; thus the populations depend approximately linearly on electron density for densities up to $\sim 10^{5} \mathrm{~cm}^{-3}$. The populations, particularly that of the $a^{4} D_{7 / 2}$ at $7955 \mathrm{~cm}^{-1}$ level, also increase monotonically with temperature under collisional excitation conditions.

For the present work we use the photoionization modeling code Cloudy (ver. 06.02; Ferland et al. 1998) to compute spectral models of the outflowing absorbing gas in QSO 2359-1241. This version of Cloudy includes the 371 level $\mathrm{Fe}^{+}$model atom of Verner et al. (1999), as well as the detailed model He I atom of Porter et al. (2005). The default version of the model He I atom ( $\mathrm{nLS}$ resolved up through principle quantum number $n=6$, plus 20 additional LS-collapsed levels lying above) was determined to be sufficient for our purposes. We assume constant total hydrogen density "clouds" of solar abundances [in particu$\operatorname{lar} \log (\mathrm{He} / \mathrm{H})=-1.0$ and $\log (\mathrm{Fe} / \mathrm{H})=-4.5498$ by number; Holweger 2001] and adopted the Mathews \& Ferland (1987) quasar spectral energy distribution (hereafter, the MF87 SED) as the incident continuum spectrum. The solar abundances are from Allende Prieto et al. (2002, 2001) for C and O, Holweger (2001) for N, Ne, Mg, Si, and Fe, and Ander \& Grevesse (1989) for the rest.

\subsection{Some General Physical Considerations}

Before we proceed to determine the physical conditions within the outflow of QSO 2359-1241, let us first examine the formation of $\mathrm{Fe}$ II and $\mathrm{He} \mathrm{I}^{*}$ within a photoionized cloud. Figure 1 (top panel) shows the ionic fractions of $\mathrm{Fe}$ II and of helium in the $\mathrm{He}^{*}{ }^{*}$ state within a representative photoionized gas cloud. Note that we plot the $\mathrm{He}^{*}$ fraction relative to that of the total iron abundance so that the comparison of the curves for $\mathrm{He} \mathrm{I}\left(2{ }^{3} S\right)$ and $\mathrm{Fe}$ II is then independent of the iron abundance. The conditions of this model cloud are: hydrogen number density $n_{\mathrm{H}}=10^{4.4} \mathrm{~cm}^{-3}$, ionization parameter given by $\log U_{\mathrm{H}}=-2.418$, solar abundances, and the MF87 SED. The particular choice of $n_{\mathrm{H}}$ and $U_{\mathrm{H}}$ will become apparent below. Here, the ionization parameter is defined as $U_{\mathrm{H}} \equiv \Phi_{\mathrm{H}} / c n_{\mathrm{H}}$, where $\Phi_{\mathrm{H}}\left(\mathrm{cm}^{-2} \mathrm{~s}^{-1}\right)$ is the hydrogen ionizing photon flux and $n_{\mathrm{H}}\left(\mathrm{cm}^{-3}\right)$ is the total hydrogen number density. The cloud is bounded by a total hydrogen column density $\log N_{\mathrm{H}}\left(\mathrm{cm}^{-2}\right)=21$. Note that the column densities of Fe II and $\mathrm{He} \mathrm{I}^{*}$ vary rapidly approaching the hydrogen ionization front [at $\log N_{\mathrm{H}}\left(\mathrm{cm}^{-2}\right) \approx 20.6$ in this model]. In particular, the $\mathrm{Fe}$ II $/ \mathrm{Fe}$ ratio rises $\sim 4$ orders of magnitude to values approaching unity within the He II zone [as indicated by the bump in He I $\left(2{ }^{3} S\right)$ ], with most of this change occurring just inside the hydrogen ionization front. For the cloud shown, $90 \%$ of the Fe II column density forms within $20 \%$ of the cloud volume lying to the left of 

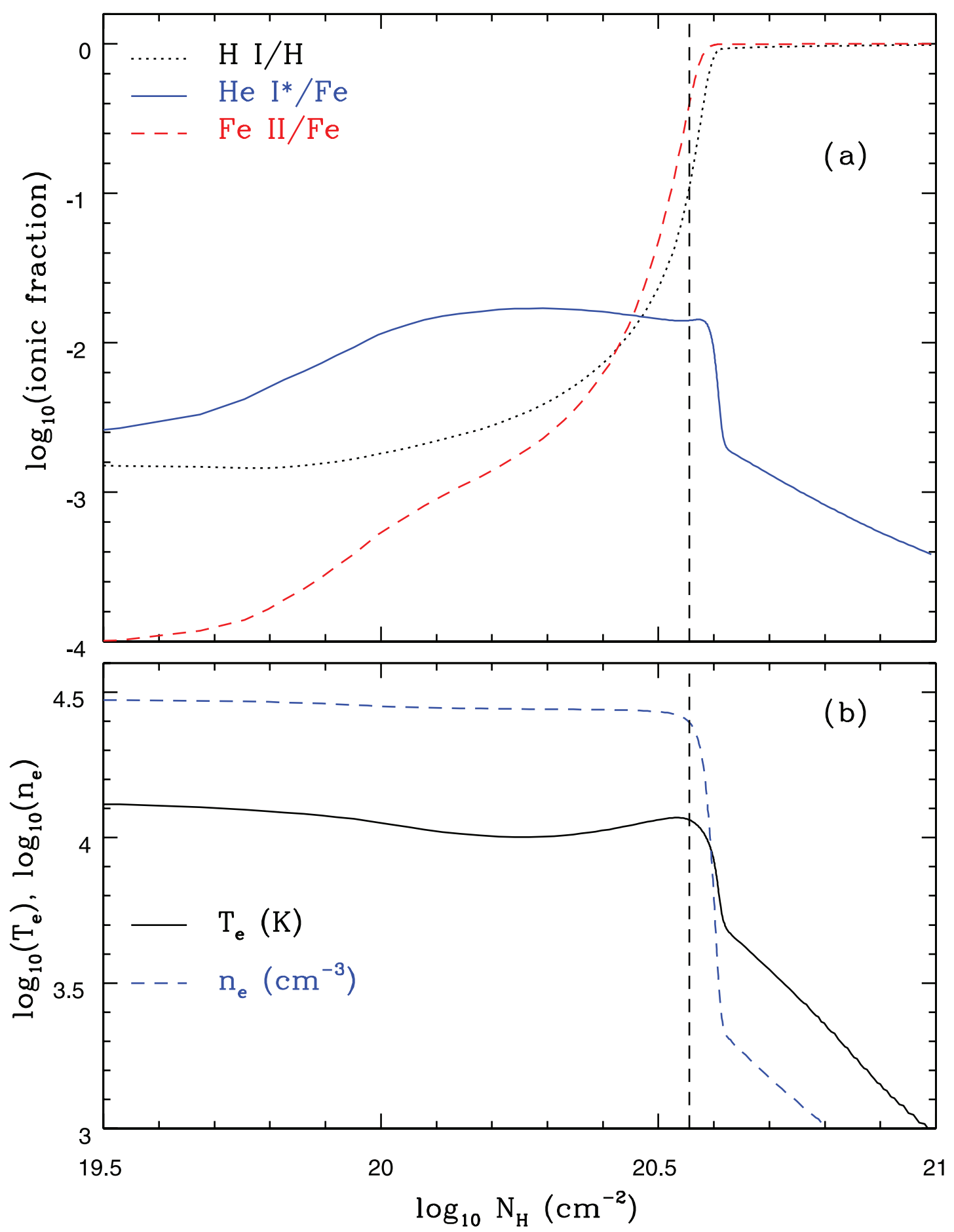

FIG. 1.- Physical structure of a photoionized cloud vs. total hydrogen column density. The parameters of the model are given in the text. Top: Fraction of neutral helium in the $2{ }^{3} \mathrm{~S}$ level and the ionic fractions of $\mathrm{H}$ I, Fe II. The He I* fraction has been normalized to the total iron abundance. Bottom: Behavior of the electron density and electron temperature. The vertical dashed line indicates the total hydrogen column density of the model to match the observed column densities of Fe II and He $I^{*}$ in QSO 2359-1241. See text for details.

the vertical dashed line. By comparison the He $\mathrm{I}^{*}$ column density increases by just $25 \%$ over the same volume of the cloud. Since nearly all of the observable Fe in forms right along the hydrogen ionization front, the model results will depend only weakly on the iron abundance.

Figure 1 (top panel) also shows that the $\mathrm{Fe}$ iI/ $\mathrm{Fe}$ ratio remains near unity behind (in the more neutral side of) the ionization front. As a consequence, the integrated $\mathrm{Fe} \mathrm{II}_{\mathrm{I}} / \mathrm{He} \mathrm{I}^{*}$ ratio continues to grow in clouds with column densities that extend beyond the hydrogen ionization front, whose column density scales as $N_{\text {ion }} \approx 10^{23} U_{\mathrm{H}} \mathrm{cm}^{-2}$. This is similarly the case for other singly ionized species of atoms with low first ionization potential $(<13.6 \mathrm{eV})$ that we measure, such as Mg II, Si II, Ca II, Mn II, and $\mathrm{Ni}$ II. However, as we show in the next section, the observed Fe II column density in QSO 2359-1241 is largely formed in the warm region on the ionized side of the hydrogen ionization front.

In Figure 1 (bottom panel) we show the run of electron temperature and electron density through the same representative cloud. Note the sharp drop in both quantities behind the hydrogen ionization front. These are accompanied by a corresponding rapid 


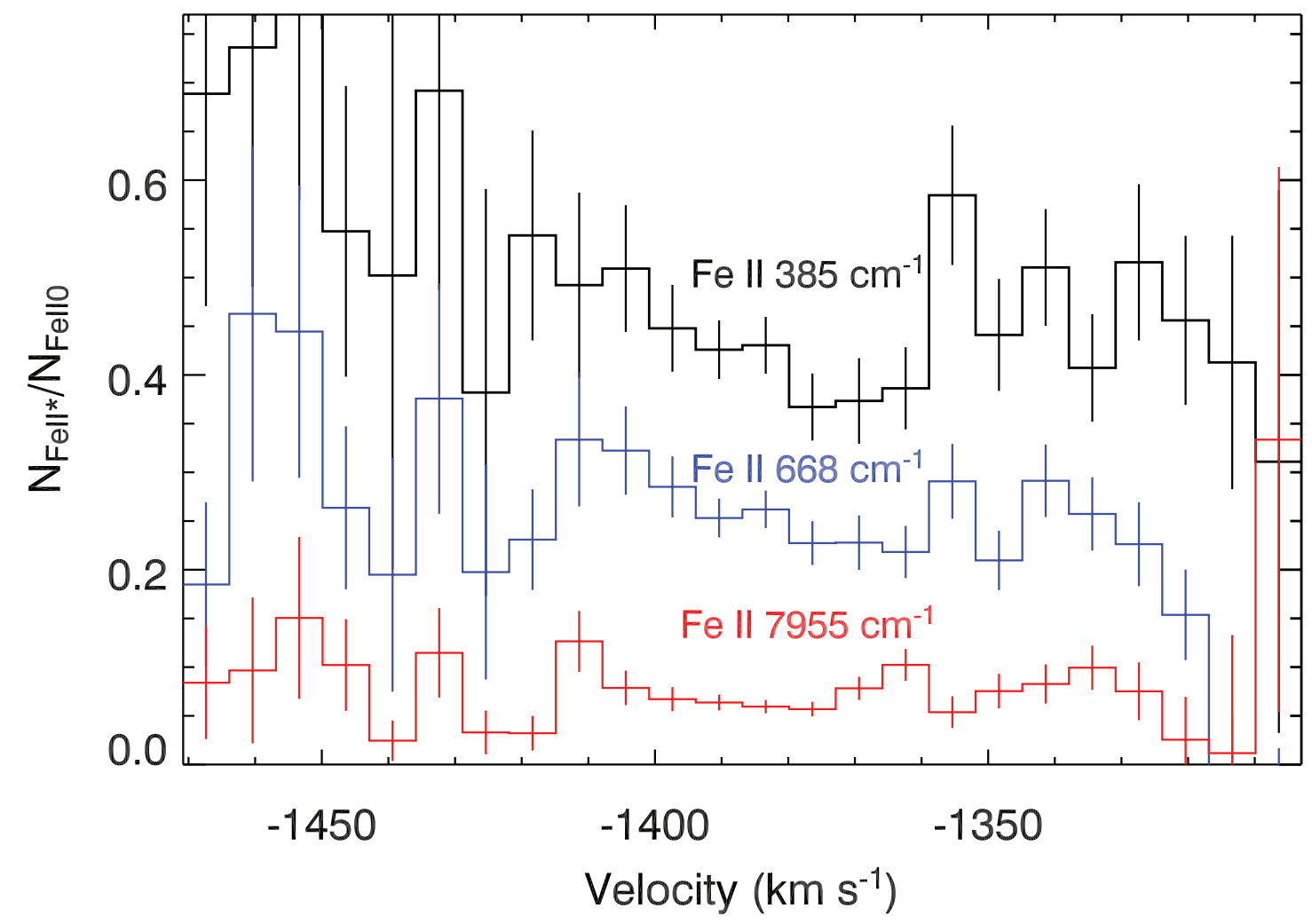

FIG. 2.-Ratios of observed column densities of excited Fe II levels to the ground level vs. velocity shift along the trough.

depopulating of excited states of the $\mathrm{Fe}$ II ion, even within the ground $a^{6} D$ term, but especially those in the $a^{4} D$ (e.g., the $7955 \mathrm{~cm}^{-1}$ level) which lie nearly $1 \mathrm{eV}$ above the ground. Thus, if absorption occurred within the "cold" neutral zone, the Fe II column density would be much greater and dominated by the resonance lines out of the ground term (especially those arising from the $0.00 \mathrm{~cm}^{-1}$ level), unlike that which is observed in the spectrum of QSO J2359-1241. We discuss this further in the next section.

Figure 1 depicts general qualitative physical properties of these types of absorption line systems, while also providing important physical constraints on our photoionization models. Furthermore, the particular model shown was found to best reproduce the observed $\mathrm{Fe}_{\text {II }}$ and $\mathrm{He}_{\mathrm{I}}{ }^{*}$ column densities. The model and the optimization technique employed to match the measured column densities are described in detail in the following section.

\subsection{Photoionization Models of the Outflow in QSO 2359-1241}

Before attempting self-consistent photoionization modeling of the absorbing gas it is useful to constrain the physical conditions of the ionized region based on the observed level-specific column densities of the $\mathrm{Fe}$ II ion as well as that of $\mathrm{He} \mathrm{I}^{*}$ (see Table 2). We use a stand-alone spectral model of Fe II (Bautista \& Pradhan 1998) to investigate the populations of the $a^{6} D_{j}$ and $a^{4} D_{j}$ levels as functions of fixed values in electron density and temperature. We find that the observed relative column densities are consistent with $\log \left(n_{e}\right)=4.4 \pm 0.1$ and $T_{e}>9000 \mathrm{~K}$. The former is constrained by the relative populations within the $a^{6} D_{j}$ ground term, while the latter (at this density) is constrained by the $a^{4} D$ $7955 \mathrm{~cm}^{-1}$ population. Further, we find no evidence for significant variations of physical conditions along the radial velocity space covered by the $\mathrm{Fe}$ II absorption troughs. In Figure 2 one sees that across a span of $\sim 100 \mathrm{~km} \mathrm{~s}^{-1}$ the column densities of the levels relative to the $0.00 \mathrm{~cm}^{-1}$ ground level are constant within the error bars, as also demonstrated by linear fits to all three curves. This implies that $n_{e}$ is similarly constant across this outflow component. As seen in Figure 1, the photoionization model solution is reached (vertical dashed line) before the values of $n_{e}$ and $T_{e}$ begin plummeting due to the $\mathrm{H}$ I ionization front. If the absorption had taken place deeper within or behind the ionization front, we should have then seen a rapid decline of the $N\left(\mathrm{Fe}\right.$ II $\left.^{*}\right) / N(\mathrm{Fe}$ II $)$ at either high or low velocity, assuming the velocity of the flow changes monotonically with radius (see the discussion regarding the physical nature of the outflow in $\S 4$ of Paper I). This is because the relative level populations within the $a^{6} D_{j}$ term are roughly linearly dependent on $n_{e}$ for $n_{e}$ well below the critical densities of the excited levels, and that of the $7955 \mathrm{~cm}^{-1}$ level also depends on $T_{e}$ (primarily via the Boltzmann factor) in our temperature range. Both effects work in the same direction: the lowering of $n_{e}$ and $T_{e}$, which occurs deep within and beyond the hydrogen ionization front (see Fig. 1), results in significantly reduced populations of the excited levels, as mentioned in $\S 3.1$. For example, in the range of electron density $\log \left(n_{e}\right)=3.4-4.4$ the excited state level populations within the $a^{6} D_{j}$ term fall by about a factor of 5 , while that of the $7955 \mathrm{~cm}^{-1}$ level falls by a decade due to electron density alone and a factor of $\sim 2$ as the temperature falls from $11,500 \mathrm{~K}$ to half that.

From these preliminary investigations we conclude that the observed $\mathrm{Fe}$ II troughs form mostly within a narrow region of the outflow near the hydrogen ionization front for which the electron density and temperature do not vary significantly, and that a hydrogen ionization front does not fully form within the flow (see Fig. 1). This then constrains the cloud column density to be $\log N_{\mathrm{H}}<20.6+\log \left(U_{\mathrm{H}} / 10^{-2.4}\right)$. This is in contrast to the findings of de Kool et al. $(2001,2002 a, 2002 b)$ in their analysis of intrinsic $\mathrm{Fe}$ II troughs in other quasars. This is an important conclusion, since the rapidly plunging values of electron density and temperature behind the hydrogen ionization front would make for more difficult modeling. 
We also use the analytic formula of Clegg (1987; see also eq. [3] of Arav et al. 2001) to estimate a ratio $n\left(2{ }^{3} S\right) / n\left(\mathrm{He}^{+}\right) \approx 5 \times$ $10^{-6}$ for the above electron density and a temperature of $10^{4} \mathrm{~K}$. Further, if we assume a solar $(\mathrm{He} / \mathrm{H})$ abundance and that $\left(\mathrm{He}^{+} /\right.$ $\mathrm{He}) \sim 0.8$ for the ionized portion of the cloud (as is typical for standard AGN SEDs), we estimate a total hydrogen column density of $N_{\mathrm{H}} \sim 2.5 \times 10^{6} N\left(2{ }^{3} S\right) \sim 10^{20.5} \mathrm{~cm}^{-2}$, given our measurement of $N\left(2{ }^{3} S\right)$ quoted in Table 2 . The substantial improvement in quality of the spectral data of QSO 2359-1241 (see also Paper I) over that presented in Arav et al. (2001) has thus already yielded much stronger constraints in the physical conditions within the outflow. We next proceed with the more detailed modeling.

In building a fully self-consistent photoionization model of the outflow of QSO 2359-1241 based on the intrinsic absorption lines, we adopt a two-step iterative photoionization modeling procedure. Using the default parameter optimization scheme built into Cloudy, the ionization parameter $U_{\mathrm{H}}$ and total hydrogen column density $N_{\mathrm{H}}$ are optimized to simultaneously match the total column density in $\mathrm{Fe}$ II and the column density in the excited-state He I*.

In the first pass we adopt a hydrogen number density, $n_{\mathrm{H}}$, equal to the electron density derived from the Fe II level specific column densities $\left(10^{4.4} \mathrm{~cm}^{-3}\right)$, and the total $\mathrm{Fe}$ II column density is estimated from the sum of the level specific column densities determined from the observations and the results of our standalone $\mathrm{Fe}$ II model atom for the above density and a temperature of $10,000 \mathrm{~K}$. The Cloudy optimizer minimizes $\chi^{2}$ between the computed and measured target column densities (with assumed equal weighting) for various values of $U_{\mathrm{H}}$ and $N_{\mathrm{H}}$. To speed up computations and since we are not concerned with the detailed $\mathrm{Fe}_{\text {II }}$ level populations during this step, the default 16 level model atom of $\mathrm{Fe}^{+}$within Cloudy is utilized during the optimization. This subset of the full 371 level model atom includes all levels up through $a^{4} P$ near $13,500 \mathrm{~cm}^{-1}$, i.e., the four lowest terms of the ion. For a model of this size only electron impact excitation followed by radiative decay through forbidden transitions is considered in the excitation. However, as verified later, radiative processes contribute little to the excitation of the Fe II levels observed.

Once a solution is found, we update the value of the total Fe II column density (this converges to be 1.80 times the sum over the six most reliably determined level-specific column densities), and then fine-tune the value of $n_{\mathrm{H}}$ by computing a grid in total hydrogen number density, spanning a decade to either side of the starting density in 0.05 dex steps, for fixed values in $U_{\mathrm{H}}$ and $N_{\mathrm{H}}$ as determined during the previous optimization step. To provide sufficient accuracy in the predictions of the level populations under study, the Cloudy models computed in the gas density grid use a 99 level model atom of $\mathrm{Fe}^{+}$(a subset of the full 371 level model atom), which includes all levels up through $50,212.8 \mathrm{~cm}^{-1}$. This model atom accounts for the dominant channels for photoexcitation by continuum radiation and other processes. Turning on the full 371 level model atom, which accounts for $\mathrm{H}$ I Ly $\alpha$ fluorescence and other processes, has virtually no impact on the level populations relevant to our study. By comparing the predicted Fe II column densities for all levels observed with the measured values in Table 2, we are able to choose the most appropriate value in total hydrogen gas number density $n_{\mathrm{H}}$. This procedure converges rapidly to a final solution. The final fit parameters are $\log n_{\mathrm{H}}=4.4$ and $\log U_{\mathrm{H}}=-2.418$, which were used for the modeled cloud shown in Figure 1, and $\log N_{\mathrm{H}}=20.556$, which is indicated by the vertical dashed line in that figure. As a further point of interest, this model's electron density-weighted temperature in the $\mathrm{Fe}^{+}$zone is $11,500 \mathrm{~K}$.
In Figure 3 we illustrate the populations of various $\mathrm{Fe}$ II levels relative to the ground level as a function of $n_{\mathrm{H}}$, assuming the final optimized values of $U_{\mathrm{H}}$ and $N_{\mathrm{H}}$ provided in the previous paragraph. We have ascertained that none of the models presented in Figure 3 have fully formed hydrogen ionization fronts within them, and so the relative level populations are indicative of the more nearly constant conditions in $n_{e}$ and $T_{e}$ self-consistently computed within the ionized portion of the cloud. ${ }^{3}$

There is very good agreement between individual determinations of the gas number density $\left(\log n_{\mathrm{H}} \approx 4.4\right)$, based on the ratios of the measured Fe II level-specific column densities ( points with error bars). The $1 \sigma$ uncertainties in the ratios are statistical only, while those in the number density are estimated by considering the range in density that results from the spread in the level ratio error bar in each direction. The robustness in the determination of the gas density is also indicated by the excellent matches between the observed and predicted level-specific Fe II column densities presented in Table 2. Altogether, these results indicate that $n_{\mathrm{H}}$ remains relatively stable through the outflow, at least within the $\mathrm{Fe}$ II zone.

For completeness, we also list in Table 2 the model predictions for all species measured in the spectra. We made no attempt to constrain the models based on these ions' measurements. With the exception of Si II, there are significant differences between predictions and observations for these species, e.g., $\mathrm{Mg}$ I and Ca II. In part these could be due to current uncertainties in their ionization balance, largely owing to errors in the dielectronic recombination rate coefficients relevant to these heavy, low-ionization species (see for example, $\S 4.3$ of Ferland et al. [1998] for a recent review, but see also Chakravorty et al. [2008], which discusses recent progress in improving these rate coefficients). Fe II is largely immune from this problem, as mentioned in $\S 3.1$, because of its strong coupling to the hydrogen ionization balance via charge transfer. On the other hand, the differences between theory and observation are also suggestive of chemical abundances that depart from solar values. In fact, supersolar metallicities are expected for gas whose origin is the center of a quasar host galaxy (see Hamann et al. 2007 for a recent review). Nearly solar $(\mathrm{Si} / \mathrm{Fe})$ and subsolar $(\mathrm{Mg} / \mathrm{Fe})$ and $(\mathrm{Ca} / \mathrm{Fe})$ together are consistent with the presence of supersolar gas metallicities in galactic chemical evolution models of massive galaxies (see, for example, Ballero et al. 2008), although these are not likely to explain the full discrepancies. Unfortunately, the paucity of observational constraints, as well as uncertainties in the atomic data for these third and, especially, fourth row elements ( $\mathrm{Ca}, \mathrm{Mn})$, prevent us from drawing definitive conclusions at the present time.

\subsection{Additional Considerations: Alternative Metallicities and SEDs}

We have found a good model for the outflow in QSO 23591241 by self-consistent optimization of the total hydrogen gas density, ionization parameter, and total hydrogen column density. In particular, for reasons already provided, we believe that our derived value in the hydrogen gas number density is robust. However, the model contains two basic assumptions concerning the gas elemental abundances (solar) and the adopted SED

\footnotetext{
3 The lower gas density clouds will be slightly cooler, all else being equal, due to the increased efficiency in the forbidden emission line cooling. The hydrogen ionization front will then occur at slightly lower column densities due to the increased recombination rate at lower temperature. Thus a larger total $\mathrm{Fe}$ II column density would be predicted for a fixed value in the cloud's total hydrogen column density. These small changes in temperature with gas density affect mainly the column density in the $0.00 \mathrm{~cm}^{-1}$ level, as well as the relative population in the $7955 \mathrm{~cm}^{-1}$ level in Fig. 3.
} 


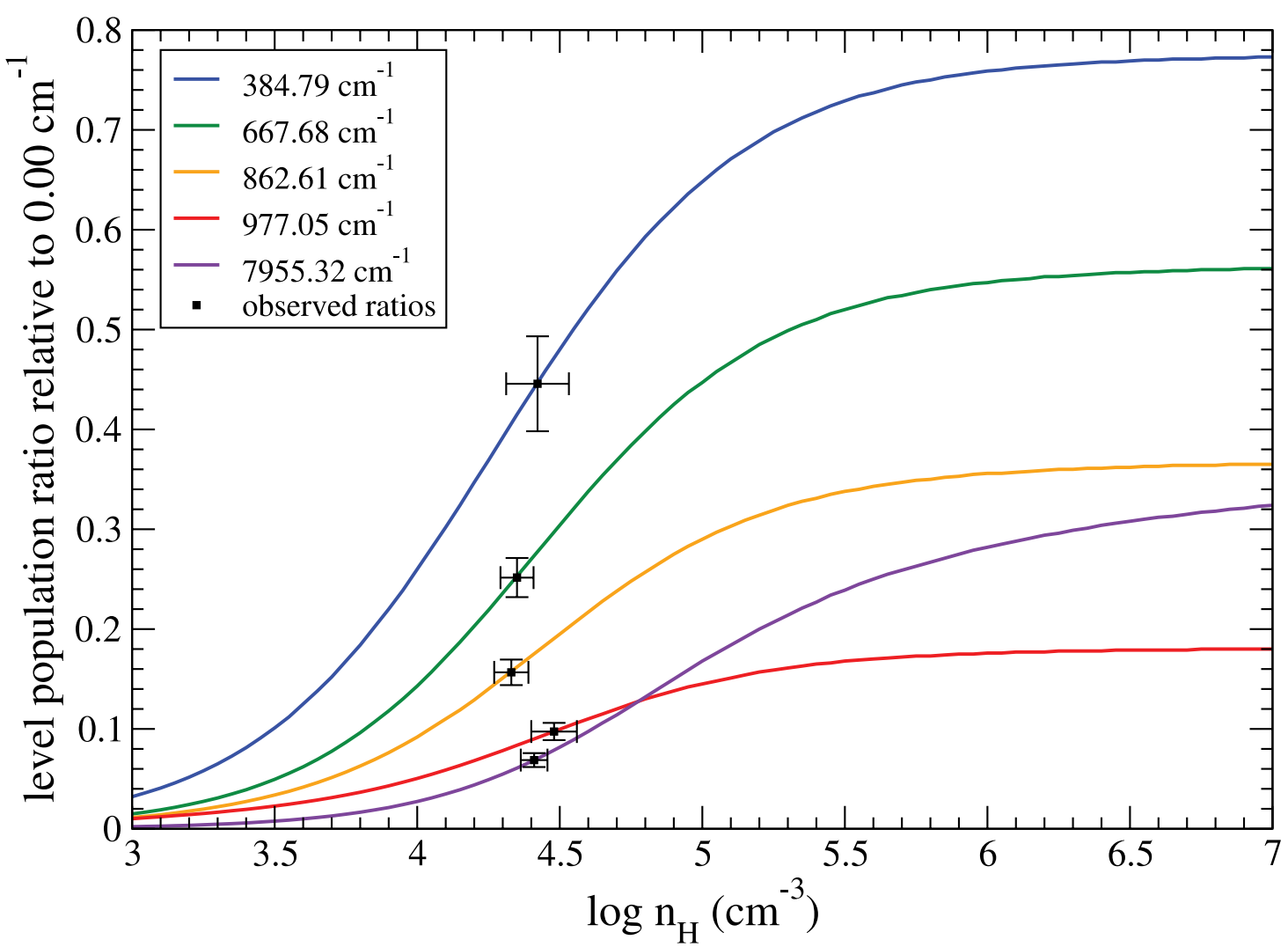

FIG. 3.-Calculated level populations of excited levels of Fe II relative to the ground level vs. hydrogen number density in the photoionization model. The points

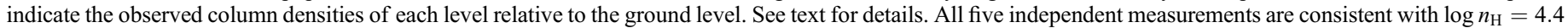
with less than $20 \%$ dispersion.

(MF87), neither of which are well constrained by the observations. Each of these assumptions independently can have important effects on the physical conditions and so on some of the inferred physical parameters, such as ionization parameter and total hydrogen column density, but in at least some cases can cancel each other under the constraints of our observations.

The assumed metallicity of the cloud affects the thermal structure of the cloud, because metals as a whole provide radiative cooling to the plasma. Thus, for a fixed SED, supersolar metallicity clouds are cooler than our optimized model, and subsolar metallicity clouds would be warmer. Thus, increasing the gas metallicity (along with $\mathrm{Fe} / \mathrm{H}$ ) also decreases the column density of the ionized zone, all else being equal, as the lower electron temperatures result in more rapid recombination rates and as the heavy elements become increasingly important opacity sources. However, we find that models with metallicities exceeding 23 times solar, for a fixed MF87 SED, are too cool ( $T$ falls below $9000 \mathrm{~K}$ ) to reproduce the observed column density ratio between the $7955 \mathrm{~cm}^{-1}$ and the ground $0.00 \mathrm{~cm}^{-1}$ levels. For larger gas metallicities, the incident SED must be correspondingly harder than the MF87 SED to push back up the electron temperature within the ionized zone. The observations of excited Fe II offer good constraints on the minimum temperature near the ionization front $(T>9000 \mathrm{~K})$, but provide no upper limit to the temperature below $20,000 \mathrm{~K}$.

The shape of the ionizing SED also affects the temperature structure of the cloud, as well as the sharpness of onset of the ionization front. The distribution of hydrogen and helium ionizing photons determines the temperature of the plasma at the illuminated face, and then the distribution of the extreme-UV and X-ray photons determines how sharply temperature and ionization drop at the ionization front and beyond. A hard SED, i.e., with a large fraction of photons in the extreme-UV and X-rays, leads to an extended ionization front, while a soft SED, with relatively more photons concentrated near $1 \mathrm{ryd}$, results in sharper declines in temperature, ionization, and electron density. We find that gas of solar abundances illuminated by an SED with a logarithmic X-ray to UV flux ratio $\alpha_{\mathrm{ox}}<-1.7$ has electron temperatures that are too low near the hydrogen ionization front to explain the observed Fe II column densities. Based on the high optical-UV luminosity of QSO 2359-1241 (Brotherton et al. 2001) and the empirical relation between $\alpha_{\mathrm{ox}}$ and $L_{\mathrm{uv}}$ in quasars (e.g., see Strateva et al. 2005), an $\alpha_{\mathrm{ox}} \approx-1.6 \pm 0.2$ might be expected. What little we do know about the X-rays in QSO 2359-1241 comes from Chandra observations of modest quality (16 photons detected) that find $\alpha_{\text {ox }} \approx-1.4 \pm 0.1$ (Brotherton et al. 2005; M. S. Brotherton 2008 , private communication; estimated error bar is statistical only), which is consistent with the above empirical relation and more importantly is the same value as that in our assumed SED (MF87). We refer the reader to Arav et al. (2001) and to Brotherton et al. (2001) for information pertaining to this object's rest-frame opticalUV spectrum.

In conclusion, the few observational constraints available do support an incident continuum SED whose average ionizing photon energy is similar to the MF87 spectrum, although there remains the possibility (we consider it unlikely) that it is substantially harder. Furthermore, the gas metallicity is unlikely to be severely subsolar. Therefore, the total column density of the absorbing gas as derived in the previous section seems secure to within a factor of 2-3.

\section{DISCUSSION AND CONCLUSIONS}

First, we compare the results of this investigation to the analysis of the Keck HIRES observations of QSO 2359-1214 by Arav et al. (2001). Using the same MF87 SED, the HIRES analysis found 
$\log N_{\mathrm{H}}=20.2$ and $\log U_{\mathrm{H}}=-2.7$, compared to $\log N_{\mathrm{H}}=20.6$ and $\log U_{\mathrm{H}}=-2.4$ for the VLT analysis. These factors of $\sim 2$ differences are mainly attributed to using apparent optical depth methods to extract the $\mathrm{Fe}$ II and $\mathrm{He}_{\mathrm{I}}^{*}$ column densities. As pointed out in Arav et al. (2001), the data was not of high enough signalto-noise ratio to permit more sophisticated analyses. Even so, Arav et al. (2001) already showed that the outflow is not shielded by a hydrogen ionization front, a result confirmed by the VLT analysis.

The important leap in diagnostic power for the VLT data came from the ability to accurately measure the population levels of the excited Fe II levels, allowing us to pinpoint the number density of the outflow to $\log \left(n_{e}\right)=10^{4.4} \mathrm{~cm}^{-3}$, to better than $20 \%$ accuracy. This is both qualitatively and quantitatively a great improvement over the lower limit of $\log \left(n_{e}\right)=10^{5} \mathrm{~cm}^{-3}$ available from the HIRES data. This result is not accidental. The main reason we invested $6.5 \mathrm{hr}$ of VLT observation on this outflow was precisely to yield a data set from which an accurate $n_{e}$ could be extracted. This determination of $n_{e}$ will allow us to determine the distance of the outflow from the central source and thus measure its mass flux and kinetic luminosity. This demonstrates the importance of taking high-quality spectra of such outflows.

Other important results arising from the measured populations of the Fe II levels are the determination of a lower limit to the temperature of the Fe II region and the realization that the absorption spectrum forms before the hydrogen ionization front, beyond which the temperature and ionization drop sharply. The temperature determination was crucial in constraining a whole family of SEDs and gas metallicities that would yield very dif- ferent temperatures in the Fe II region, consequently allowing for a more secure determination of the total gas column density and ionization parameter. That the Fe II absorption occurs in a region of nearly constant conditions before the hydrogen ionization front is a key to being able to model the absorption spectrum.

Photoionization modeling allowed us to reproduce quite well the observed $\mathrm{Fe}$ II and $\mathrm{He}_{\mathrm{I}}{ }^{*}$ column densities in the main component, $e$, of the quasar outflow absorption spectrum. Reiterating, we found $\log N_{\mathrm{H}} \approx 20.6$ and $\log U_{\mathrm{H}} \approx-2.4$. The dominant error bars to these values come from the uncertainties in the assumed SED and gas metallicities and come to $\sim 0.3$ dex.

Given the above gas density and ionization parameter, an estimate to an unobscured incident bolometric luminosity of $\sim 4.7 \times$ $10^{47} \mathrm{ergs}^{-1}$ based on the intrinsic reddening correction in Brotherton et al. $(2001,2005)$, and a standard cosmology $\left(H_{0}=\right.$ $\left.70 \mathrm{~km} \mathrm{~s}^{-1} \mathrm{Mpc}^{-1}, \Omega_{\Lambda}=0.70, \Omega_{m}=0.30\right)$, we estimate a distance of component $e$ of the outflow from the central continuum source of $\sim 3 \mathrm{kpc}$. In a future paper we will similarly determine the physical conditions in the weaker, lower velocity outflow components $a-d$, as well as the distances and estimates of the kinetic luminosities for all components in the outflow, which are important to AGN feedback scenarios of galaxy evolution.

We acknowledge support from NSF grant AST 0507772 and from NASA LTSA grant NAG5-12867. We also would like to thank the anonymous referee for his or her helpful comments and suggestions.
Allende Prieto, C., Lambert, D. L., \& Asplund, M. 2001, ApJ, 556, L63 . 2002, ApJ, 573, L137

Anders, E., \& Grevesse, N. 1989, Geochim. Cosmochim. Acta, 53, 197

Arav, N., Barlow, T. A., Laor, A., \& Blandford, R. D. 1997, MNRAS, 288, 1015

Arav, N., Becker, R. H., Laurent-Muehleisen, S. A., Gregg, M. D., White, R. L., Brotherton, M. S., \& de Kool, M. 1999a, ApJ, 524, 566

Arav, N., Brotherton, M. S., Becker, R. H., Gregg, M. D., White, R. L., Price, T., \& Hack, W. 2001, ApJ, 546, 140

Arav, N., Kaastra, J., Kriss, G. A., Korista, K. T., Gabel, J., \& Proga, D. 2005, ApJ, 620, 665

Arav, N., Kaastra, J., Steenbrugge, K., Brinkman, B., Edelson, R., Korista, K. T., \& de Kool, M. 2003, ApJ, 590, 174

Arav, N., Korista, K. T., \& de Kool, M. 2002, ApJ, 566, 699

Arav, N., Korista, K. T., de Kool, M., Junkkarinen, V. T., \& Begelman, M. C. 1999b, ApJ, 516, 27

Arav, N., Moe, M., Costantini, E., Korista, K. T., Benn, C., \& Ellison, S. 2008, ApJ, 681, 954

Arav, N., et al. 2007, ApJ, 658, 829

Ballero, S. K., Matteucci, F., Ciotti, L., Calura, F., \& Padovani, P. 2008, A\&A, 478,335

Bautista, M. A. 2004, A\&A, 420, 763

Bautista, M. A., \& Pradhan, A. K. 1998, ApJ, 492, 650

Brotherton, M. S., Arav, N., Becker, R. H., Tran, H. D., Gregg, M. D., White, R. L., Laurent-Muehleisen, S. A., \& Hack, W. 2001, ApJ, 546, 134

Brotherton, M. S., Laurent-Muehleisen, S. A., Becker, R. H., Gregg, M. D., Telis, G., White, R. L., \& Shang, Z. 2005, AJ, 130, 2006

Cattaneo et al. 2005, MNRAS, 364, 407

Chakravorty, S., Kembhavi, A. K., Elvis, M., Ferland, G., \& Badnell, N. R. 2008, MNRAS, 384, L24

Clegg, R. E. S. 1987, MNRAS, 229, 31

de Kool, M., Arav, N., Becker, R. H., Gregg, M. D., White, R. L., LaurentMuehleisen, S. A., Price, T., \& Korista, K. T. 2001, ApJ, 548, 609

de Kool, M., Becker, R. H., Arav, N., Gregg, M. D., \& White, R. L. 2002a, ApJ, 570, 514 de Kool, M., Becker, R. H., Gregg, M. D., White, R. L., \& Arav, N. 2002b, ApJ, 567, 58

de Kool, M., Korista, K. T., \& Arav, N. 2002c, ApJ, 580, 54

Ferland, G. J., Korista, K. T., Verner, D. A., Ferguson, J. W., Kingdon, J. B., \& Verner, E. M. 1998, PASP, 110, 761

Gabel, J. R., et al. 2005, ApJ, 623, 85

Hamann, F., Warner, C., Dietrich, M., \& Ferland, G. 2007, ASP Conf. Ser. 373, The Central Engine of Active Galactic Nuclei, ed. L. C. Ho \& J.-M. Wang (San Francisco: ASP), 653

Holweger, H. 2001, in AIP Conf. Proc. 598, Joint SOHO/ACE Workshop Solar and Galactic Composition, ed. R. F. Wimmer-Schweingruber (Melville: AIP), 23

Hopkins, P. F., Hernquist, L., Cox, T. J., Di Matteo, T., Robertson, B., \& Springel, V. 2006, ApJS, 163, 1

King, A. 2003, ApJ, 596, L27

Kurucz, K. T., \& Bell, B. 1995, Atomic Line Data, Kurucz CD ROM 23 (Cambridge, MA: SAO)

Mathews, W. G., \& Ferland, G. J. 1987, ApJ, 323, 456

Morton, D. C. 2003, ApJS, 149, 205

Osterbrock, D. E., \& Ferland, G. J. 2006, Astrophysics of Gaseous Nebulae and Active Galactic Nuclei (2nd. ed.; Sausalito: Univ. Sci. Books)

Porter, R. L., Bauman, R. P., Ferland, G. J., \& MacAdam, K. B. 2005, ApJ, 622, L73

Scott, J. E., Kriss, G. A., Brotherton, M., Green, R. F., Hutchings, J., Shull, J. M., \& Zheng, W. 2004, ApJ, 615, 135

Silk, J., \& Rees, M. J. 1998, A\&A, 331, L1S

Strateva, I. V., Brandt, W. N., Schneider, D. P., Vanden Berk, D. G., \& Vignali, C. 2005, AJ, 130, 387

Telfer, R. C., Kriss, G. A., Zheng, W., Davidsen, A. F., \& Green, R. F. 1998, ApJ, 509, 132

Verner, E. M., Verner, D. A., Korista, K. T., Ferguson, J. W., Hamann, F., \& Ferland, G. J. 1999, ApJS, 120, 101

Wampler, E. J., Chugai, N. N., \& Petitjean, P. 1995, ApJ, 443, 586 